\title{
KLOE-2 results and perspectives on hadron physics
}

\section{Marcin Berłowski on behalf of KLOE-2 collaboration}

National Centre for Nuclear Research

Pasteura 7, Warsaw, Poland

E-mail: Marcin.Berlowski@ncbj.gov.pl

Recent results from the KLOE/KLOE-2 experiments on hadron physics are presented. The KLOE-2 project continues and extends the program of KLOE in the field of discrete symmetry tests, light meson spectroscopy, dark matter searches etc. The KLOE-2 collaboration finished the data taking campaign in March of 2018 collecting $5.5 \mathrm{fb}^{-1}$ which is with the previous KLOE data set of $2.5 \mathrm{fb}^{-1}$ the world's biggest sample taken at the phi meson mass $(1020 \mathrm{MeV})$ in any $\mathrm{e}^{+} \mathrm{e}^{-}$collider. The new measurement of the Dalitz plot of $\eta \rightarrow \pi^{\circ} \pi^{+} \pi^{-}$and the light quark masses ratio determination are presented. The measurements of the Transition Form Factors of $\phi \rightarrow \eta \mathrm{e}^{+} \mathrm{e}^{-}$ and $\phi \rightarrow \pi^{\circ} \mathrm{e}^{+} \mathrm{e}^{-}$are also reported. Finally the preliminary results from analysis looking for a signal from $\eta \rightarrow \pi^{\circ} \gamma \gamma$ and $\eta \rightarrow \pi^{+} \pi^{-}$decay channels are discussed.

The 9th International workshop on Chiral Dynamics - CD2018

17-21 September, 2018

Durham, NC, USA 


\section{Introduction}

KLOE and KLOE-2 detectors operated at DAFNE [1], an electron-positron collider $\phi$-factory working at $\sqrt{\mathrm{s}}_{\mathrm{s}} \approx 1020 \mathrm{MeV}$. The produced particles are recorded by the detector, consisting of a large $(\mathrm{R}=2 \mathrm{~m})$ cylindrical drift chamber [2], surrounded by an electromagnetic calorimeter made of lead scintillating-fibers covering $98 \%$ of $4 \pi$ [3]. Both of them are inside a magnetic field of about $0.5 \mathrm{~T}$. In 2008 a new interaction scheme was introduced in order to increase the machine luminosity [4]. The KLOE-2 detector was upgraded with a new interaction region constituted by an inner cylindrical triple-GEM 4 layer tracker [5] for larger track acceptance at low $p_{t}{ }^{\text {'s }} \mathrm{s}$ and to improve the charged vertex reconstruction, new calorimeters in the beamline region $[6,7]$ to improve acceptance for particles emitted at small polar angles and a scintillator stripes tagger placed after the first bending magnet of the machine designed for the search of $\gamma \gamma$ physics [8].

KLOE was taking data in the years 2001-2006, collecting $2.5 \mathrm{fb}^{-1}$ of $\phi$ decays. The KLOE-2 dataset was collected in 2014-2018 and amounts to $5.5 \mathrm{fb}^{-1}$. KLOE and KLOE-2 have provided the largest sample of $\mathrm{e}^{+} \mathrm{e}^{-} \rightarrow \phi$ events collected in $\mathrm{e}^{+} \mathrm{e}^{-}$collider, corresponding to about $2.4 \times 10^{10}$ produced $\phi$ mesons.

\section{2. $\quad \eta \rightarrow \pi^{\circ} \pi^{+} \pi^{-}$Dalitz plot distribution}

The isospin violating $\eta \rightarrow \pi^{+} \pi^{-} \pi^{\circ}$ decay can be used as a test bench in Chiral Perturbation Theory (ChPT), for a low-energy QCD effective field theory, and as a precise probe of the u-d quark mass difference. The decay amplitude is proportional to light quark mass ratio (in ChPT $\Gamma$ $\left.\sim \mathrm{Q}^{-4}\right)$ :

$$
Q^{2} \equiv \frac{m_{s}^{2}-\widehat{m}^{2}}{m_{d}^{2}-m_{u}^{2}}, \text { where } \widehat{m}=\frac{1}{2}\left(m_{d}+m_{u}\right)
$$

Eq. 1 is valid up to NLO ChPT. After correcting for the electromagnetic effects at the lowest order by using a combination of kaon and pion masses, a $Q$ value $=24.2$ is obtained. The theoretical calculations show a very slow convergence of the ChPT effective field theory decay widths [9]: $\Gamma_{\mathrm{LO}}=66 \mathrm{eV}$ and $\Gamma_{\mathrm{NLO}}=160-210 \mathrm{eV}$. Those values can be compared to the experimental results $\Gamma_{\exp }=300 \pm 12 \mathrm{eV}$ showing discrepancy that could originate from higher order contributions to the decay amplitude or from corrections to the $\mathrm{Q}$ value. In order to provide input for the dispersive calculations and to clarify the discrepancy between theory [1012] and the experiments [13-17], higher statistic measurements are required.

The Dalitz plot density in the $\eta$ meson rest frame can be parametrized using Taylor expansion around $\mathrm{X}=\mathrm{Y}=0$ :

$$
|A(X, Y)|^{2} \approx 1+\mathrm{a} Y+\mathrm{b} Y^{2}+\mathrm{c} X+\mathrm{d} X^{2}+\mathrm{e} X Y+\mathrm{f} Y^{3}+\mathrm{g} X^{2} Y+\cdots
$$

where $X=\sqrt{3} \frac{T_{\pi+}-T_{\pi-}}{Q}, Y=\frac{3 T_{\pi^{\circ}}}{Q}-1, Q=T_{\pi+}+T_{\pi-}+T_{\pi^{\circ}} . T_{\pi^{\circ \pm}}$ are the kinetic energies of the final state $\pi^{\circ \pm}$ particles. C-invariance excludes terms with odd powers of $X$. The parameters $a, b$, ... in Eq. 2 are going to be extracted by fitting the experimental Dalitz plot distribution. 

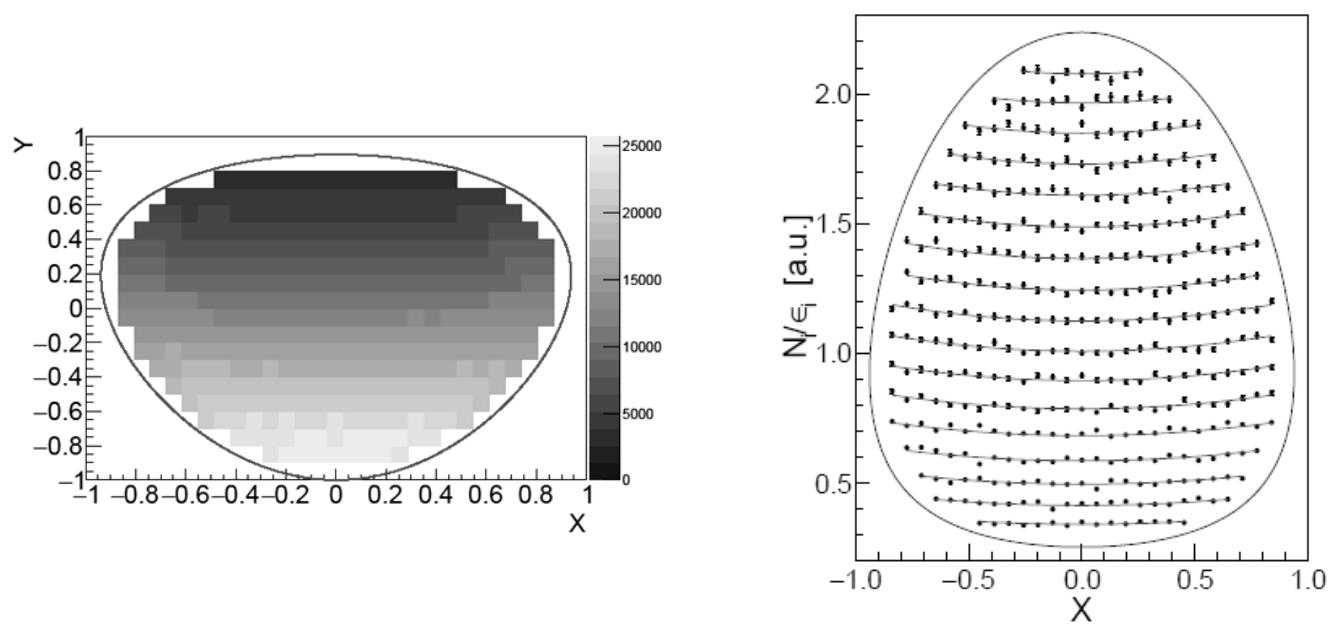

Fig. 1. Left: background-subtracted Dalitz plot. Right: acceptance corrected Dalitz plot together with the result of the fit. Only statistical uncertainties are shown.

The analysis of the $\eta \rightarrow \pi^{+} \pi^{-} \pi^{\circ}$ decay was based on $1.7 \mathrm{fb}^{-1}$ data collected by KLOE detector [18]. Events containing two oppositely charged tracks and three neutral clusters originating from the interaction point were selected. The highest-energy photon coming from the 2-body $\phi$ decay $\phi \rightarrow \eta \gamma$, was identified by requiring $\mathrm{E}_{\gamma}>250 \mathrm{MeV}$. Discrimination against electron contamination from Bhabha scattering were achieved by the time-of-flight measurement of the clusters connected to the charged tracks in the electromagnetic calorimeter. Additional cuts have been applied in order to reduce the remaining background: a) on the angle between $\pi^{+}$or $\pi^{-}$and the closest photon from $\pi^{\circ}$ decay, b) on the angle between neutral clusters in the $\pi^{\circ}$ rest frame, and c) on the reconstructed $\pi^{\circ}$ invariant mass squared. Signal selection efficiency of $37.6 \%$ was obtained with signal to background ratio $S / N=133$ in the final data sample with less than $1 \%$ of residual background. The Dalitz plot distribution was constructed using 31 and 20 bins for $X$ and $Y$ respectively and consisted of $\sim 4.7 \cdot 10^{6}$ events in total. The bin width of the Dalitz plot was determined by the resolution in $X$ and $Y$ and the number of events in each of the bins yielding to the 371 bins in the final plot. Left panel of Fig. 1 shows data distribution after background subtraction and the acceptance corrected data are presented on the right panel of Fig. 1.

The final results for the Dalitz plot parameters [18], including the systematic effects, are:

$$
\begin{gathered}
a=-1.095 \pm 0.003_{-0.002}^{+0.003} \\
b=+0.145 \pm 0.003 \pm 0.005 \\
d=+0.081 \pm 0.003_{-0.005}^{+0.006} \\
f=+0.141 \pm 0.007_{-0.008}^{+0.007} \\
g=-0.044 \pm 0.009_{-0.013}^{+0.012}
\end{gathered}
$$

These measurements are in agreement with the previous KLOE results [15] improving the accuracy by a factor of three. This is the first statistically significant measurement of the $Y^{2} X$ term (g parameter). In addition, tests of $\mathrm{C}$ conservation in the decay were performed by studying asymmetries for the unbinned data. All the results are consistent with zero at the $10^{-4}$ level. For example the asymmetry between kinetic energies of positively and negatively charged pions, $\mathrm{A}_{\mathrm{LR}}$, was found to be:

$$
A_{L R}=\left(-5.0 \pm 4.5_{-11}^{+5.0}\right) \times 10^{-4}
$$


The new KLOE results, at the moment the most precise available, are being used as an input in dispersive analyses, for example in determination of the quark mass ratio $\mathrm{Q}=22.0 \pm 0.7$ by the Bern group [19].

\section{Meson Transition Form Factors}

Transition Form Factors (TFFs) describe mesons coupling to photons and provide description of their nature and structure. TFFs are fundamental measurements in hadron physics, useful for effective Lagrangians of low-energy QCD based on Chiral Perturbation Theory (ChPT) [20, 21] and for studies of the transition regime from soft, non-pQCD, to pQCD hard interactions. TFFs were recently becoming once again interesting due to the g-2 discrepancy. While the main contribution to the hadronic contribution to muon anomaly is the vacuum polarization, the second one in order of relevance comes from the hadronic Light-by-Light (HLbL) scattering, for which the leading contribution is the single pseudoscalar meson exchange. The measurement of TFFs for the $\eta$ and the $\pi^{\circ}$ mesons for the time-like region is obtained by studying Dalitz decays or $\mathrm{V} \rightarrow \mathrm{P} \gamma^{*}$ transition processes, while meson production in $\gamma \gamma$ interaction via $\mathrm{e}^{+} \mathrm{e}^{-} \rightarrow$ $\mathrm{e}^{+} \mathrm{e}^{-} \gamma^{*} \gamma^{*} \rightarrow \mathrm{e}^{+} \mathrm{e}^{-\mathrm{P}}$ gives the coupling to space-like photons. Nevertheless any experimental information on the TFFs, for space-like and time-like $\mathrm{q}^{2}$, can help in constraining the models used in the calculations [22].

In conversion decays, $\mathrm{A} \rightarrow \mathrm{B} \gamma^{*} \rightarrow \mathrm{Bl}^{+} 1^{-}$, the radiated photon is virtual and the squared dilepton invariant mass, $\mathrm{M}^{2}{ }_{\mathrm{ll}}$, corresponds to the photon 4-momentum transferred, $\mathrm{q}^{2}$. The probability of having a lepton pair of given invariant mass is determined by the electromagnetic dynamical structure of the transition $\mathrm{A} \rightarrow \mathrm{B} \gamma^{*}$. The slope of the transition form factor, $\mathrm{b}_{\mathrm{AB}}$, is defined as:

$$
\left.b_{A B} \equiv \frac{d F_{A B}\left(q^{2}\right)}{d q^{2}}\right|_{q^{2}=0}
$$

In the Vector Meson Dominance (VMD) model, the transition form factor is parametrized as:

\section{1 $\phi \rightarrow \eta \mathrm{e}^{+} \mathrm{e}^{-}$}

$$
F_{A B}\left(q^{2}\right)=\frac{1}{1-\frac{q^{2}}{\Lambda_{A B}^{2}}} \rightarrow b_{A B} \approx \Lambda_{A B}^{-2}
$$

The decay chain $\phi \rightarrow \eta \mathrm{e}^{+} \mathrm{e}^{-}$, where $\eta \rightarrow 3 \pi^{\circ}$, has been performed by analysing a data sample of about $1.7 \mathrm{fb}^{-1}$. The preselection creteria consists in the request to have just 6 prompt photons and two oppositely charged tracks close to the interaction region. Further selection constrains have been implemented in order to achieve a low background contamination (at the level of $3 \%$ ). Finally around 30 thousand candidate events survived all the selection conditions and the most prominent contamination consists in $\phi \rightarrow \mathrm{K}_{\mathrm{S}} \mathrm{K}_{\mathrm{L}} \rightarrow \pi^{+} \pi^{-} 3 \pi^{\circ}$ channel concentrating at high $\mathrm{e}^{+} \mathrm{e}^{-}$masses. After bin-by-bin background subtraction on $\mathrm{M}_{\mathrm{ee}}$ spectrum (Fig. 2 left panel) the BR measurement is [23]:

$$
\mathrm{BR}\left(\phi \rightarrow \eta \mathrm{e}^{+} \mathrm{e}^{-}\right)=(1.075 \pm 0.007 \pm 0.038) \times 10^{-4},
$$

where the systematics are dominated by the uncertainty on the normalization, $\phi$ production cross section and $\eta \rightarrow 3 \pi^{\circ}$ branching ratio.

The transition form factor $F_{\phi \eta}\left(q^{2}\right)$ was obtained from a fit to $M_{e e}$ spectrum with the function: 
$\frac{1}{\Gamma(\phi \rightarrow \eta \gamma)} \frac{d \Gamma\left(\phi \rightarrow \eta e^{+} e^{-}\right)}{d q^{2}}=\frac{\alpha}{3 \pi} \frac{\left|F_{\phi \eta}\left(q^{2}\right)\right|^{2}}{q^{2}} \sqrt{1-\frac{4 M^{2}}{q^{2}}}\left(1+\frac{2 M^{2}}{q^{2}}\right)\left[\left(1+\frac{q^{2}}{M_{\phi}^{2}-M_{\eta}^{2}}\right)^{2}-\frac{4 M_{\phi}^{2} q^{2}}{\left(M_{\phi}^{2}-M_{\eta}^{2}\right)^{2}}\right]^{3 / 2}$

The comparison with data is show on Fig. 2, center panel. The comparison of $\left|F_{\phi \eta}\left(q^{2}\right)\right|^{2}$ as a function of $\mathrm{M}_{\mathrm{ee}}$ and predictions from VMD are shown on Fig. 2, right panel.
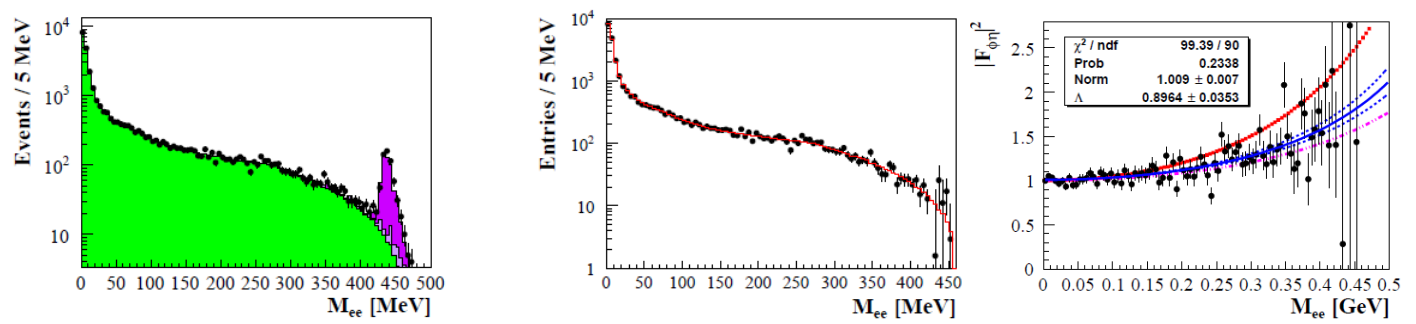

Fig. 2. $M_{e e}$ spectrum at the end of the analysis chain, points are data, green $\phi \rightarrow \eta e^{+} e^{-}$signal, purple $\phi \rightarrow K_{S} K_{L} \rightarrow \pi^{+} \pi^{-} 3 \pi^{\circ}$ background (left). The same spectrum after bin-by-bin background subtraction with superimosed fit line in red (center). Fit of $\left|F_{\phi \eta}\right|^{2}$ as a function of $M_{e e}$ in blue and predictions from VMD in pink and from [20] in red (right).

The same spectrum as for the fit were used in order to obtain the TFF slope parameter $b_{\phi \eta}$ by dividing the histogram bin-by-bin by Monte Carlo generated distribution with $\mathrm{F}_{\phi \eta}=1$. The result of the measurement is:

$$
b_{\phi \eta}=\left(0.17 \pm 0.10_{-0.11}^{+0.07}\right) \mathrm{GeV}^{-2}
$$

The $\operatorname{BR}\left(\phi \rightarrow \eta e^{+} e^{-}\right)$value, and the transition form factor slope $b_{\phi \eta}$ measurements are in the agreement with the VMD predictions [24], and with the SND and CMD-2 results [25, 26] and their precision have been improved by a factor of five.

\section{$4.2 \phi \rightarrow \pi^{\circ} \mathbf{e}^{+} \mathbf{e}^{-}$}

The $\phi \rightarrow \pi^{\circ} \mathrm{e}^{+} \mathrm{e}^{-}$decay channel [27] was identified by looking for two neutral clusters in the calorimeter with reconstructed mass close to the $\pi^{\circ}$ meson and two tracks of opposite charge in the drift chamber close to the beam interaction region. The separation of electrons from pions and muons was done using time of flight technique and reduction of photon conversion background by looking at the mass of pairs measured at the position of the beam pipe. The only remaining backgrounds were radiative Bhabha scattering process and the leftovers of $\phi \rightarrow \pi^{\circ} \gamma$ with photon conversion into $\mathrm{e}^{+} \mathrm{e}^{-}$pair. At the end of the analysis chain around 15 thousand candidate events were left with background contamination of $\sim 35 \%$, which corresponds to about 9500 signal events (see Fig. 3, left panel). The analysis was limited for $\sqrt{ }^{2} \leq 700 \mathrm{MeV}$ due to high decrease of efficiency for the highest values of the momentum transfer. The $\mathrm{M}_{\mathrm{ee}}$ spectrum was bin-by-bin background subtracted taking into account MC derived efficiency, which led to the branching fraction measurement of [27]:

$$
B R\left(\phi \rightarrow \pi^{\circ} e^{+} e^{-} ; \sqrt{q^{2}}<700 \mathrm{MeV}\right)=\left(1.19 \pm 0.05_{-0.10}^{+0.05}\right) \times 10^{-5}
$$

The first quoted error is the statistical including the normalization uncertainty, while the second is the systematical, including variations of analysis cuts as well as the uncertainty on background fitting procedure. The branching fraction can be extended to the full kinematical range using the form factor from [28] giving: 


$$
B R\left(\phi \rightarrow \pi^{\circ} e^{+} e^{-}\right)=\left(1.35 \pm 0.05_{-0.10}^{+0.05}\right) \times 10^{-5}
$$

The value obtained significantly improves the results obtained by SND and CMD-2 [29, 30] experiments, and is in agreement with theoretical predictions.

Fig. 3, right panel, shows the results of $\left|\mathrm{F}_{\phi \pi^{\circ}}\left(\mathrm{q}^{2}\right)\right|^{2}$ compared with theoretical predictions. The best agreement is obtained with the Unconstrained Resonant Chiral Theory, where NA60 data have been used as an input to the model [28]. From the fit the value of TFF slope parameter is:

$$
b_{\phi \pi^{\circ}}=(2.02 \pm 0.11) \mathrm{GeV}^{-2}
$$

This value can be compared with the expectations from one pole approximation $b_{\phi \pi^{\circ}}=\mathrm{M}_{\phi}^{-2}=$ $\sim 1 \mathrm{GeV}^{-2}$ and dispersive analysis result from ref. [21] $\mathrm{b}_{\phi \pi^{\circ}}=(2.52-2.68) \mathrm{GeV}^{-2}$.
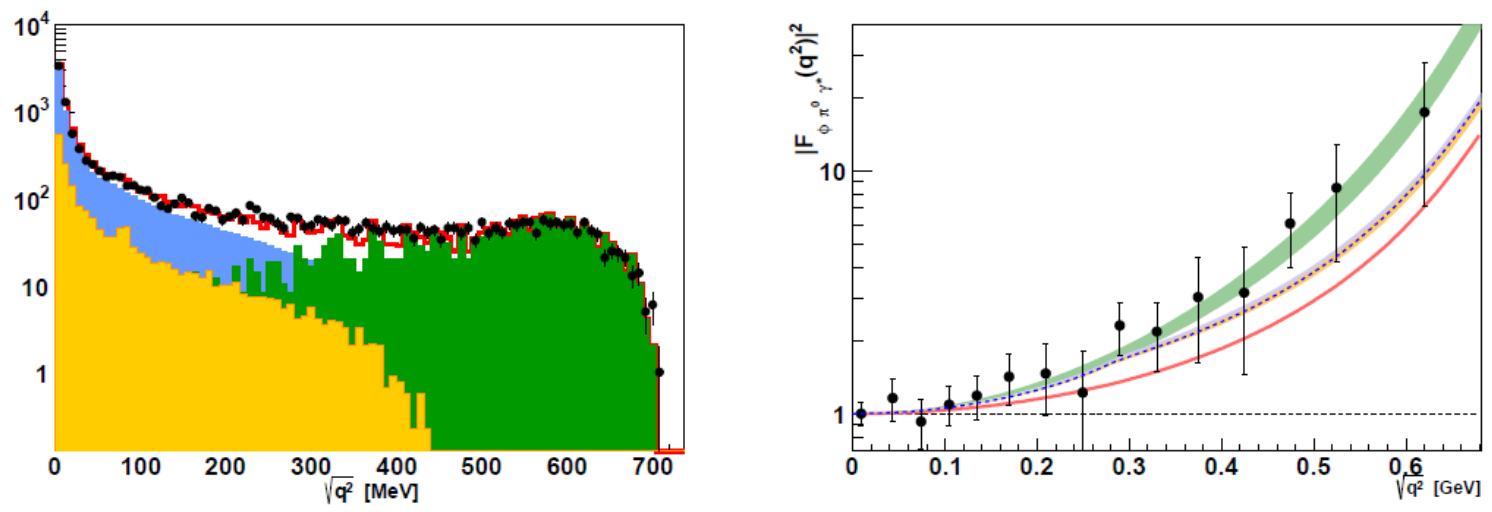

Fig. 3. Left: Data-MC comparison at the end of analysis chain for the invariant mass of $e^{+} e^{-}$. The black points are data, solid red line is the sum of all shown MC components: signal (blue), $\phi \rightarrow \pi^{\circ} \gamma($ orange), radiative Bhabha scattering (green). Right: Comparison between the measurement of $\left|F_{\phi \pi}\left(q^{2}\right)\right|^{2}($ black points), theoretical predictions of ref [21](orange band) and ref [28](green band) and one-pole VMD model (solid red line).

\section{5. $\eta \rightarrow \pi^{\circ} \gamma \gamma$}

The $\eta \rightarrow \pi^{\circ} \gamma \gamma$ decay is very interesting from the experimental and the theoretical point of view. The theory made impressive progress since last 40 years when the first very large values for the branching ratio were obtained [31]. The experimental value of this branching fraction [32-34] decreased with time with the increase of machine luminosities and the available statistics of the $\eta$ meson samples produced, showing that the main issue in the measurement of the branching fraction is the correct background estimation. Moreover this "golden mode" decay is of particular interest in ChPT, due to the fact that the chiral Lagrangian terms start at $\mathrm{O}\left(\mathrm{p}^{4}\right)$ which are then highly suppressed and the dominating contribution from $\mathrm{O}\left(\mathrm{p}^{6}\right)$ terms can be used in order to test $\chi \mathrm{PT}$ predictions. The other interesting subject concerning this decay is the search for possible contribution from leptophobic dark matter mediator which couples mainly to quarks. In the most basic model the coupling is to baryon number via kinetic mixing term $\varepsilon$ [35]. In the case of such process we expect to see a sharp resonance peak in the $\pi^{\circ} \gamma$ invariant mass spectrum.

At KLOE this decay proceeds via $\phi \rightarrow \eta \gamma$, therefore we are looking for the final state with 5 prompt (aligned in time) photons and no charged tracks. The main background processes are radiative decays of the $\phi$ meson with either $a_{0}$ or $f_{0}$ in the final state, direct production of $\omega$ meson $\mathrm{e}^{+} \mathrm{e}^{-} \rightarrow \omega\left(\rightarrow \pi^{\circ} \gamma\right) \pi^{\circ}$ and $\eta$ meson decay into $3 \pi^{\circ}$, when some of the photons are lost and/or merged into another clusters. 
The KLOE analysis from 2006 was based on a data sample of $450 \mathrm{pb}^{-1}$. The number of signal events was estimated to be around 70. By using MC the efficiency of about $5 \%$ and the $\eta \rightarrow 3 \pi^{\circ}$ normalization channel have been evaluated, then we obtain the preliminary result for the $\eta \rightarrow \pi^{\circ} \gamma \gamma$ branching ratio [36]:

$$
\operatorname{BR}\left(\eta \rightarrow \pi^{\circ} \gamma \gamma\right)=\left(8.4 \pm 2.7_{\text {stat }} \pm 1.4_{\text {syst }}\right) \times 10^{-5}
$$

This result is lower by $2 \sigma$ then $\chi \mathrm{PT}$ predictions as well as the $\mathrm{CB} @ \mathrm{AGS} / \mathrm{CB} @ \mathrm{MAMI}$ measurements [37, 38].

Recently the reanalysis of the channel started using full KLOE data sample of $1.7 \mathrm{fb}^{-1}$. It uses the kinematic fits with different particle mass constrains in order to distinguish backgrounds and signal. Fig. 4 shows the preliminary distribution of $\mathbf{M}_{4 \gamma}$ before and after applying constrained kinematic fits. We have a very good data-MC description and we are currently working with signal to background at the level of $20 \%$, were the main contribution comes from $\eta$ meson decay into $3 \pi^{\circ}$ which can mimic the signal when photons are lost or merged with other cluster. Multivariate data analysis method is currently under tuning to reduce the background coming from the merged clusters in $\eta \rightarrow 3 \pi^{\circ}$ background channel.

By using full KLOE/KLOE-2 data sample of $\sim 8 \mathrm{fb}^{-1}$ and assuming $5 \%$ analysis efficiency statistics around 1000 events and a better background reduction from $\eta \rightarrow 3 \pi^{\circ}$ thanks to the increased detector's acceptance for photons are expected.

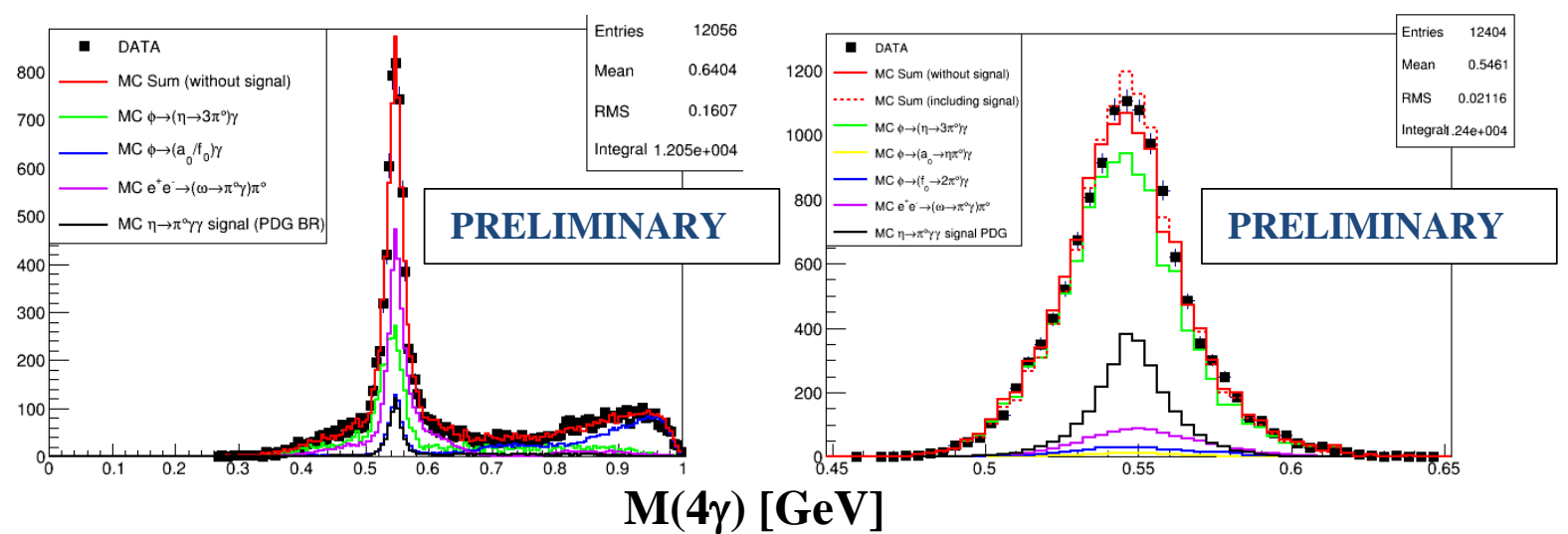

Fig. 4. Invariant mass of $4 \gamma$ before (left) and after (right) applying constrained kinematic fits selection: data (points), simulated signal with current PDG branching ratio in black, $\eta \rightarrow 3 \pi^{\circ}$ in green, MC sum in red.

\section{6. $\eta \rightarrow \pi^{+} \pi^{-}$}

The $\eta \rightarrow \pi^{+} \pi^{-}$decay violates both P and CP invariance. In the Standard Model it can proceed only via the weak interaction with a branching ratio of the order of $10^{-27}$. If we introduce a $\mathrm{CP}$ violating term in the $\mathrm{QCD}$, its $\mathrm{BR}$ enhances to $10^{-17}$, while allowing $\mathrm{CP}$ violation in the extended Higgs sector we can get BR at order of $10^{-15}$ [39]. Any observation of larger branching ratio would indicate a new source of $\mathrm{CP}$ violation in the strong interactions. The best limit for the branching ratio comes from KLOE collaboration based on $350 \mathrm{pb}^{-1}$ data sample [40]:

$$
\operatorname{BR}\left(\eta \rightarrow \pi^{+} \pi^{-}\right)<1.3 \times 10^{-5} @ 90 \% \text { C.L. }
$$

Recently also a new measurement came from LHCb collaboration [41]:

$$
\operatorname{BR}\left(\eta \rightarrow \pi^{+} \pi^{-}\right)<1.6 \times 10^{-5} @ 90 \% \text { C.L. }
$$


A new KLOE analysis, using more statistics $\left(\mathrm{L}_{\text {int }} \sim 1.7 \mathrm{fb}^{-1}\right)$, is carried over to update the upper limit. The $\eta$ production is tagged by monochromatic photon of energy of $365 \mathrm{MeV}$. The analysis uses time-of-flight technique and track mass measurement $\left(\mathrm{T}_{\mathrm{M}}\right.$ is the mass value that satisfies equation below) as independent sources to separate pions from other charged particles:

$$
\left|\vec{p}_{\phi}-\vec{p}_{1}-\vec{p}_{2}\right|=E_{\phi}-\sqrt{p_{1}^{2}+T_{M}^{2}}-\sqrt{p_{2}^{2}+T_{M}^{2}}
$$

In the equation above $\mathrm{E}_{\phi}$ is phi meson energy and $\mathrm{p}_{\mathrm{i}}$ corresponds to pions and the $\phi$ meson momenta. The remaining source of background is the continuum coming from $\gamma \pi \pi$ and $\rho \pi$. There is no visible event excess in the $\mathrm{M}_{\pi \pi}$ spectrum in the $\eta$ region and by using fit with polynomial function in addition to MC signal shape (see Fig. 5 right panel) we can extract the preliminary branching ratio upper limit:

$$
\operatorname{BR}\left(\eta \rightarrow \pi^{+} \pi^{-}\right)<6.3 \times 10^{-6} @ 90 \% \text { C.L. }
$$

Currently the momentum-dependent correction for tracks and estimation of systematic effects is ongoing. A new approach to the extraction of upper limit by only fitting the background region ( $\eta$ sidebands) is under testing.

By using all KLOE/KLOE-2 data $\left(8 \mathrm{fb}^{-1}\right)$ and assuming the same acceptance and efficiency an upper limit of $2.7 \times 10^{-6} @ 90 \%$ C.L. on BR is expected.
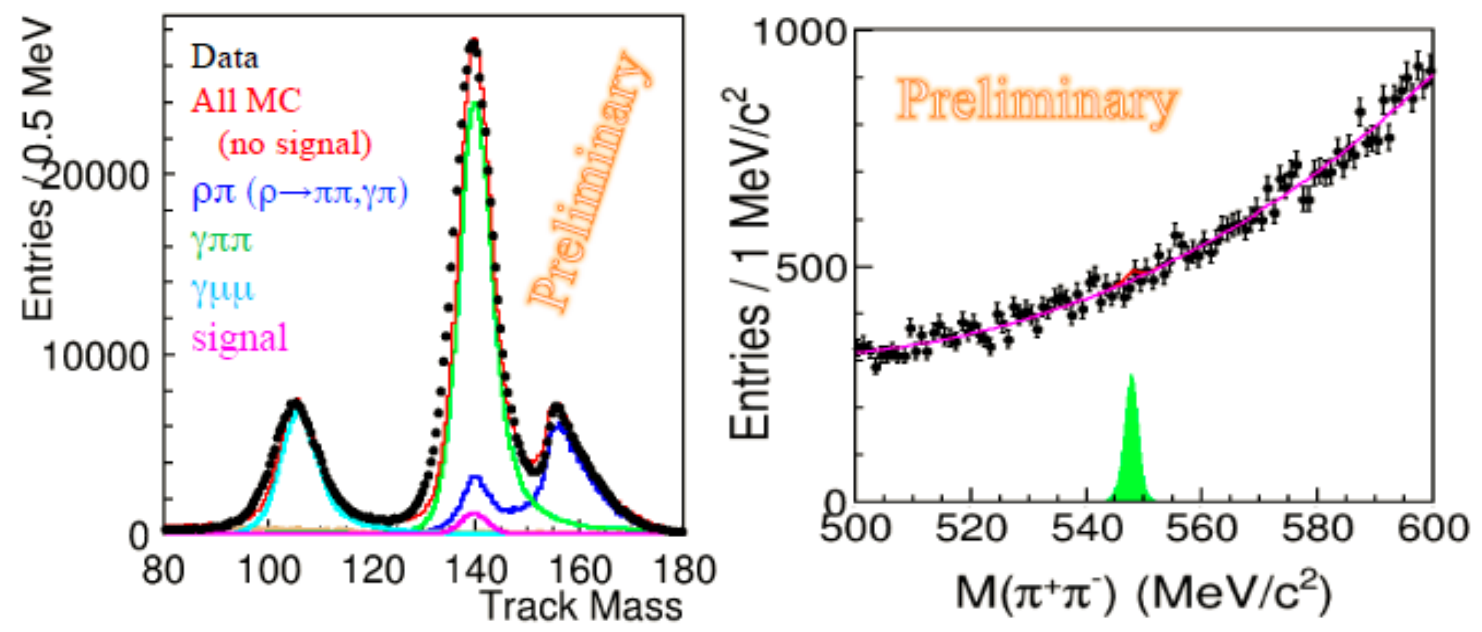

Fig. 5. On the left: the track mass variable used to distinguish between pions and electrons or muons using time of flight technique. On the right: invariant mass spectrum of $\pi^{+} \pi^{-}$system, expected signal shape in green filled histogram, pink line shows the result of the fit to the data (points).

\section{Summary and perspectives}

The KLOE-2 collaboration continues to analyze the high statistics data samples collected during the first period of KLOE data-taking, to perform detailed measurements in hadron physics. The precision study of the light meson properties will continue with increased statistics collected in the KLOE-2 campaign, which ended in March of 2018 with an integrated luminosity of $5.5 \mathrm{fb}^{-1}$. Moreover the new KLOE-2 data benefits of an upgraded detector (new calorimeters, inner tracker and lepton taggers) with a larger acceptance (from $21^{\circ}$ to $10^{\circ}$ ), a better charged particles tracking and vertex reconstruction, and the access to the $\gamma \gamma$ physics. 
Future investigations in hadron physics by using KLOE-2 data can be summarized in the following list:

- Meson transition form factors, improving $\phi \rightarrow \eta \mathrm{e}^{+} \mathrm{e}^{-}$and $\phi \rightarrow \pi^{\circ} \mathrm{e}^{+} \mathrm{e}^{-}$, and measuring $\phi \rightarrow \eta \mu^{+} \mu^{-}, \phi \rightarrow \eta \pi^{+} \pi^{-}$, and $\mathrm{e}^{+} \mathrm{e}^{-} \rightarrow \pi^{0} \gamma \gamma_{\mathrm{ISR}}$

- the rare $\eta$ decays $\left(\eta \rightarrow \pi^{\circ} \gamma \gamma, \eta \rightarrow 4\right.$ charged tracks, limits on $\eta \rightarrow \gamma \gamma \gamma, \pi^{+} \pi^{-}, \pi^{\circ} \pi^{\circ}, \pi^{\circ} \pi^{\circ} \gamma$ );

- the $\gamma \gamma$ process $\mathrm{e}^{+} \mathrm{e}^{-} \rightarrow \mathrm{e}^{+} \mathrm{e}^{-} \gamma^{*} \gamma^{*} \rightarrow \mathrm{e}^{+} \mathrm{e}^{-} \pi^{\circ}, \pi^{\circ}$ radiative width and the TFFs at low- $\mathrm{q}^{2}$;

- searches for dark bosons, improving the existing limits and looking for the new hypothetical particles, like leptophobic gauge bosons or axion-like particles.

Acknowledgements We warmly thank our former KLOE colleagues for the access to the data collected during the KLOE data taking campaign. We thank the DAFNE team for their efforts in maintaining low background running conditions and their collaboration during all data taking. We want to thank our technical staff: G.F. Fortugno and F. Sborzacchi for their dedication in ensuring efficient operation of the KLOE computing facilities; M. Anelli for his continuous attention to the gas system and detector safety; A. Balla, M. Gatta, G. Corradi and G. Papalino for electronics maintenance; C. Piscitelli for his help during major maintenance periods. This work was supported in part by the Polish National Science Centre through the Grant No. 2017/26/M/ST2/00697.

\section{References}

[1] A. Gallo et al., DAFNE status report, Conf. Proc. C060626, 604-606 (2006)

[2] M. Adinolfi et al., The tracking detector of the KLOE experiment, Nucl. Instrum. Meth. A 488, 5173 (2002)

[3] M. Adinolfi et al., The KLOE electromagnetic calorimeter, Nucl. Instrum. Meth. A 482, 364-386 (2002)

[4] G. Amelino-Camelia et al., Physics with the KLOE-2 experiment at the upgraded DAfNE, Eur. Phys. J. C 68, 619-681 (2010)

[5] A. Balla et al., Status of the Cylindrical-GEM project for the KLOE-2 Inner Tracker, Nucl. Instrum. Meth. A 628, 194-198 (2011)

[6] M. Cordelli et al., CCALT: A Crystal CALorimeter with Timing for the KLOE-2 upgrade, Nucl. Instrum. Meth. A 718, 81-82 (2013)

[7] M. Cordelli et al., QCALT: A Tile calorimeter for KLOE-2 experiment, Nucl. Instrum. Meth. A 617, 105-106 (2010)

[8] D. Babusci et al., The KLOE-2 High Energy Tagger Detector, arXiv:1206.0680 [physics.ins-det]

[9] K. Kampf, M. Knecht, J. Novotný, M. Zdráhal, Analytical dispersive construction of $\eta \rightarrow 3 \pi$ amplitude: First order in isospin breaking, Phys. Rev. D 84, 114015 (2011)

[10] G. Colangelo, S. Lanz, H. Leutwyler, E. Passemar, Determination of the light quark masses from $\eta \rightarrow 3 \pi$, PoS EPS-HEP2011 304 (2011)

[11] P. Guo et al., Three-body final state interaction in $\eta \rightarrow 3 \pi$, Phys. Rev. D 92, 054016 (2015)

[12] M. Gormley et al., Experimental Determination of the Dalitz-Plot Distribution of the Decays $\eta \rightarrow \pi^{+} \pi^{-} \pi^{\circ}$ and $\eta \rightarrow \pi^{+} \pi^{-} \gamma$, and the Branching Ratio $\eta \rightarrow \pi^{+} \pi^{-} \gamma / \eta \rightarrow \pi^{+} \pi^{-} \pi^{\circ}$, Phys. Rev. D 2, 501505 (1970)

[13] J. Layter et al., Study of dalitz-plot distributions of the decays $\eta \rightarrow \pi^{+} \pi^{-} \pi^{\circ}$ and $\eta \rightarrow \pi^{+} \pi^{-} \gamma$, Phys. Rev. D 7, 2565-2568 (1973)

[14] CRYSTAL BARREL collaboration, A. Abele et al., Momentum dependence of the decay $\eta \rightarrow \pi^{+} \pi^{-} \pi^{\circ}$, Phys. Lett. B 417, 197-201 (1998)

[15] KLOE collaboration, F. Ambrosino et al., Determination of $\eta \rightarrow \pi^{+} \pi^{-} \pi^{\circ}$ Dalitz plot slopes and asymmetries with the KLOE detector, JHEP 0805, 006 (2008) 
[16] WASA-at-COSY collaboration, P. Adlarson et al., Measurement of the $\eta \rightarrow \pi^{+} \pi^{-} \pi^{\circ}$ Dalitz plot distribution, Phys. Rev. C 90, 045207 (2014)

[17] BESIII collaboration, M. Ablikim et al., Measurement of the matrix elements for the decays $\eta \rightarrow \pi^{+} \pi^{-} \pi^{\circ}$ and $\eta / \eta^{\prime} \rightarrow \pi^{\circ} \pi^{\circ} \pi^{\circ}$ Phys. Rev. D 92, 012014 (2015)

[18] KLOE-2 collaboration, A. Anastasi et al., Precision measurement of the $\eta \rightarrow \pi^{+} \pi^{-} \pi^{\circ}$ Dalitz plot distribution with the KLOE detector, JHEP 1605, 019 (2016)

[19] G. Colangelo, S. Lanz, H. Leutwyler, E. Passemar, $\eta \rightarrow 3 \pi$ : Study of the Dalitz Plot and Extraction of the Quark Mass Ratio Q, Phys. Rev. Lett. 118, 022001 (2017)

[20] C. Terschlusen, S. Leupold, Electromagnetic Transition Form Factors of Mesons, Prog. Part. Nucl. Phys. 67, 401 (2012)

[21] S.P. Schneider, B. Kubis, F. Niecknig, $\omega \rightarrow \pi \gamma^{*}$ and $\phi \rightarrow \pi^{q} \gamma^{*}$ transition form factors in dispersion theory, Phys. Rev. D 86, 054013 (2012)

[22] F. Jegerlehner and A. Nyffeler, The Muon g-2, Phys. Rept. 477, 1-110 (2009)

[23] KLOE-2 collaboration, A.Anastasi et al., Study of Dalitz decay $\phi \rightarrow \eta e^{+} e^{-}$with KLOE detector, Phys. Lett. B 742, 1-6 (2015)

[24] A. Faessler, C. Fuchs, M.I. Krivoruchenko, Dilepton spectra from decays of light unflavored mesons, Phys. Rev. C 61, 035206 (2000)

[25] CMD-2 collaboration, R.R. Akhmetshin et al., Study of conversion decays $\phi \rightarrow \eta e^{+} e^{-}, \eta \rightarrow e^{+} e^{-} \gamma$ and $\eta \rightarrow \pi^{+} \pi^{-} \mathrm{e}^{+} \mathrm{e}^{-}$at CMD-2, Phys. Lett. B 501, 191 (2001)

[26] SND collaboration, M.N. Achasov et al., Study of Conversion Decays $\phi \rightarrow \eta e^{+} e^{-}$and $\eta \rightarrow \gamma e^{+} e^{-}$in the Experiment with SND Detector at the VEPP-2M Collider, Phys. Lett. B 504, 275 (2001)

[27] KLOE-2 collaboration, A.Anastasi et al.,Measurement of the $\phi \rightarrow \pi^{\circ} e^{+} e^{-}$transition form factor with the KLOE detector, Phys. Lett. B 757, 362-367 (2016)

[28] S. Ivashyn, Vector to pseudoscalar meson radiative transitions in chiral theory with resonances, Prob. Atomic Sci. Technol. 2012N1, 179 (2012)

[29] SND collaboration, M.N. Achasov, et al., Measurement of the $\phi \rightarrow \pi^{\circ} e^{+} e^{-}$decay probability, JETP Lett. 75, 449 (2002)

[30] CMD-2 collaboration, R.R. Akhmetshin, et al., Observation of the conversion decay $\phi \rightarrow \pi^{\circ} e^{+} e^{-}$at CMD-2, Phys. Lett. B 503, 237 (2001)

[31] L. Ametller, J. Bijnens, A. Bramon, F. Cornet, Chiral perturbation theory for $\eta \rightarrow \pi q \gamma$, Phys. Lett. B 276, 185 (1992)

[32] D. Alde et al., Neutral decays of the $\eta$-meson, Z. Phys. C 25, 225 (1984)

[33] S. Prakhov et al., Measurement of the branching ratio for $\eta \rightarrow \pi q \gamma$ decay, Phys. Rev. C 72, 025201 (2005)

[34] N. Knecht et al., Relative branching ratio of the $\eta \rightarrow \pi q \gamma$ decay channel, Phys. Lett. B 589, 14 (2004)

[35] S. Tulin, New weakly coupled forces hidden in low-energy QCD, Phys. Rev. D 89, 114008 (2014)

[36] B. Di Micco et al., The $\eta \rightarrow \pi q \gamma, \eta / \eta$ ' mixing angle and status of $\eta$ mass measurement at KLOE, Acta Phys. Slov. 56, 403 (2006)

[37] Crystal Ball collaboration at the AGS, S. Prakhov et al., Measurement of the invariant-mass spectrum for the two photons from the $\eta \rightarrow \pi 0 \gamma \gamma$ decay, Phys. Rev. C 78, 015206 (2008)

[38] A2 collaboration at MAMI, B.M.K. Nefkens et al., New measurement of the rare decay $\eta \rightarrow \pi 0 \gamma \gamma$ with the Crystal Ball/TAPS detectors at the Mainz Microtron, Phys. Rev. C 90, 025206 (2014)

[39] C. Jarlskog, E. Shabalin, On Searches for CP, T, CPT and C Violation in Flavour-Changing and Flavour-Conserving Interactions, Phys. Scripta T 99, 23 (2002)

[40] KLOE collaboration, F. Ambrosino et al., Upper limit on the $\eta \rightarrow \pi^{+} \pi^{-}$branching ratio with the KLOE detector, Phys. Lett. B 606, 276-280 (2005)

[41] LHCb collaboration, R. Aaij et al., Search for the CP-violating strong decays $\eta \rightarrow \pi^{+} \pi^{-}$and $\eta^{\prime}(958) \rightarrow \pi^{+} \pi^{-}$, Phys. Lett. B 764, 233 (2017) 